\title{
Mechanical Model of Traditional Thai Massage for Integrated Healthcare
}

\author{
Salinee Rattanaphan* and Panya Srichandr \\ Integrated Product Design and Manufacturing Program, School of Energy, \\ Environment and Materials \\ King Mongkut's University of Technology Thonburi, Bangkok, Thailand
}

Submitted November 2014. Accepted for publication February 2015.

\begin{abstract}
In this study, a mechanical model was developed, aiming to provide standardized and programmable traditional Thai massage (TTM) therapy to patients. The TTM was modeled and integrated into a mechanical hand $(\mathrm{MH})$ system, and a prototype massage chair was built and tested for user satisfaction. Three fundamental principles of Thai massage were integrated: pull, press, and pin. Based on these principles, the mechanics of Thai massage was studied and a mathematical model was developed to describe the dynamics and conditions for the design and prototyping of an MH. On average, it was found that users were satisfied with the treatment and felt that the treatment was similar to that performed by human hands. According to the interview results, users indicated that they were likely to utilize the $\mathrm{MH}$ as an alternative to traditional massage. Therefore, integrated TTM with an MH may help healthcare providers deliver standardized, programmable massage therapy to patients as opposed to variable, inconsistent human massage.
\end{abstract}

Keywords: Thai massage, mechanical model, massage technique

\section{INTRODUCTION}

Traditional Thai massage (TTM) is very popular, not only in Thailand but also in other countries [1]. It is a branch of traditional Thai medicine and one of the methods used in the primary health care system in Thailand [2]. Currently, its utilization in alternative medical treatments for many conditions has increasingly revealed that TTM on back muscles promotes relaxation, reduces stress, and increases parasympathetic activity in patients with back pain; it also increases blood circulation, decreases blood pressure, decreases heart rate, reduces pain, and promotes relaxation and is the most frequently-used alternative treatment for back pain in Thailand [3, 4]. In addition, massaging relaxes the muscles, helps release tension, reduces stress and anxiety, and ultimately provides a state of wellbeing [5]. For instance, a common and painful musculoskeletal disorder called myofascial trigger point (MTrPs) is one of the most frequently diagnosed conditions in the United States and it is a source of back pain [6]. It

*Corresponding author: Salinee Rattanaphan, Integrated Product Design and Manufacturing Program, School of Energy, Environment and Materials, King Mongkut's University of Technology Thonburi, 126 Pracha-utid Road, Bangmod, Toongkru, Bangkok 10140, Thailand. Phone: (662) 427-0039. Fax: (662) 848-1267. Email: salinee.r@gmail.com. Other author: panya.sri@kmutt.ac.th, yodpanyaa@gmail.com. 
is a challenge for health professionals to find effective treatments for it; however, massage appears to relieve this pain [1] because it releases endorphins that increase the pain threshold by closing the gate of pain at the spinal cord level [7]. Traditional Thai massage may help increase pain-relieving neurotransmitters such as serotonin, which influences the decrease of pain intensity. Additionally, a decrease in muscle tension is the likely result of increased relaxation response and broad reduction in the defense-arousal response [3]. As a potential pain-reducing mechanism, traditional Thai massage can activate proprioceptors (spindle cell and Golgi tendon organs) in targeted muscles, so it can reduce muscle spasm and adhesion between different tissues [4].

Ancient Thai massage, following Indian Ayurvedic practices, identifies pressure points and energy lines that can be stimulated to prevent and cure illness and increase well-being. Under this form of Thai massage, the "sip sen" or the "ten lines" of energy flowing in the human body maintain good health. If the energy or "wind" is blocked in a sen line, the body will be susceptible to illness. Six of these sen lines cross the body on the back and have several pressure points in the lower and upper back. Massaging these lines and points is thought to alleviate headaches, stiff neck, sinus problems, colds, abdominal pain, restless legs, urinary tract disorders, back pain and knee pain [8]. The lines are not physical lines that can be located scientifically in the body; they are rather concepts from ancient Thai massage that masseurs/masseuses use to guide them in the delivery of the massage.

In traditional Thai massage, the masseur/masseuse uses the palm, thumb, and fingers with pressure to massage parts of the body for the treatment of muscle tension or pain $[8,9]$. Professional masseurs/masseuses more or less have the same level of expertise; however, the delivery of the massage therapy is perceived by users to vary from masseur/masseuse to masseur/masseuse. The massage technique might be the same but variations in the masseur's/masseuse's hand size, hand strength, arm pressure, and even height can lead to variations in the massage. These variations are then perceived by the patient as a different style of massage, which in some instances can lead to patient dissatisfaction as well as difference in therapeutic efficacy. In order to eliminate these variations in style, in this work, we studied the techniques of massage and developed a mechanical model that provides a standard delivery of Thai massage.

Historically, two main types of Thai massage have developed: (1) the royal Thai massage, mainly for the royal family and members of the court; and (2) the typical Thai massage for people in general $[10,11]$. The Ratchsamnak method, or "royal style", provides a deep muscle massage exerting slow, increasing pressure with only the use of fingers, thumbs, and palms [12], while in the more typical Thai massage, the masseur/masseuse uses not only fingers and hands, but also arms, elbows, and sometimes knees and feet. In addition, royal Thai style massage exerts pressure on the body in a rhythmic fashion [13]. In this case, the masseur/masseuse needs to adjust the palm pressure and the angle and direction of the motion; these are referred to as the fundamental actions of Thai massage and they are denoted as: (1) pull, (2) press and (3) pin, with a temporal application of approximately 10-15 seconds for short periods and from 15 to a maximum of 45 seconds for long periods [14]. 
In this paper we used the Thai massage principles to develop a mathematical model for the design of a mechanical hand (MH) with the three actions of the royal Thai style massage. The integrated $\mathrm{MH}$ will only simulate the royal Thai massage with the use of palm, fingers, and hand motion for the massage of the lower and upper back of the body.

\section{METHOD}

\subsection{Background - Mechanics of the Royal Thai Massage}

In order to develop an $\mathrm{MH}$ that performs the royal Thai massage, we needed to understand the actual movements of the palm and fingers of the masseur/masseuse during massage. In a previous publication [15], we described the three P's: pull, press and pin (the fundamental massage actions in royal Thai massage $[16,17]$ ).

In the pull action, the masseur/masseuse uses his/her palm and fingers to pull the muscle of the person in the area to be massaged, reducing pressure as the palms lose touch with the muscle (see Figure 1 from $a$ to $c$, where the motion is from left to right).

(a)

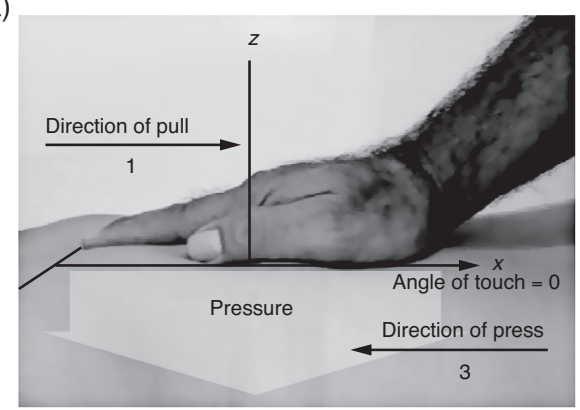

(b)

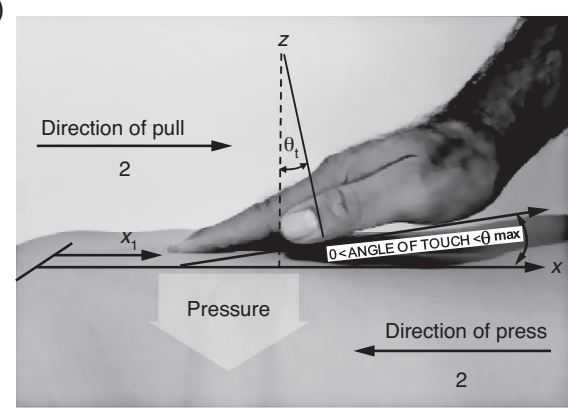

(c)

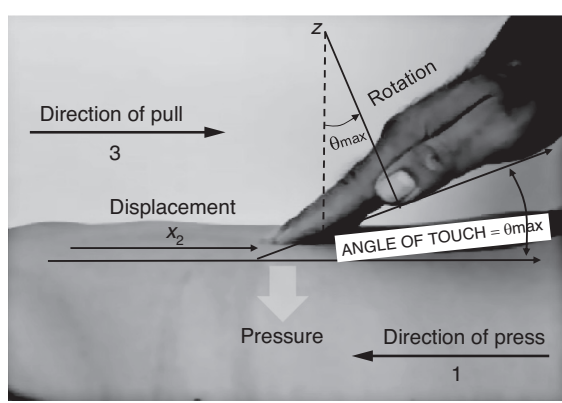

Figure 1. Pull and press motions in royal Thai massage. With palm and fingers pressing the muscle, the pull action starts with the pulling of the muscle in the $x$-direction (a). As the pull continues, the palm begins to lift from the back, making an angle $\theta_{t}$ with the surface of the muscle (b) and continues until only the tips of the fingers touch the muscle (c). The overall motion displays the translation and rotation of the hand. The press action is the reverse of the pull; it starts with the tips of the fingers touching the muscle as in (c) and slides forward in the negative $x$-direction, exerting more pressure as in (b) and ending with the full palm pressing the muscle as in (a), with no angle between the palm and the muscle. 
The masseur/masseuse then reverses the direction for the press action and gradually applies higher pressure using fingers and palms until stopping with the palm resting on the muscle (see Figure 1 in reverse order from $c$ to $a$, with motion from right to left). In the pin step, the masseur/masseuse presses the muscle with his/her fingers to a comfortable level for a brief period of time. The three steps are then repeated on the same or on a different area of the body. These massage actions were the key dynamics in the design of the MH for traditional Thai massage.

We studied the mechanics and developed a model as if the massage were performed on a patient resting horizontally, mainly for practical purposes, keeping in mind that the user will be resting on the backrest of a chair. The pressure of the massage will depend on the reclining angle of the backrest. This is a passive force system, as opposed to an $\mathrm{MH}$ exerting force on the back of the user resting on a bed, as in an active force system.

\subsection{The Pull Action}

In the pull action, the fingers and palms of the masseur/masseuse exert pressure on the muscle, and as the muscle is pulled and the hands change angle the pressure is reduced. Figure 1 shows that the pull action is a compound motion consisting of rotation and translation of the hand. The rotation is determined by the angle formed between the palm and the surface of the muscle; this angle is called the "angle of touch" and is denoted by $\theta_{t}$. $\theta_{t}$ changes from zero to a maximum angle denoted as $\theta_{\max }$, and the translation component is described by the net displacement of the hand in the $x$-direction. Thus, at the start of the pull, say at time $t=0$, the hand rests flat at $x=0$ with $\theta_{t}=0$, and at some final point in time $t=t_{f}$, the displacement of the hand is at the final displacement $x_{f}=x_{2}$ with $\theta_{t}=\theta_{\max }$ (Figure 1.a). At $x_{f}$, only the tips of the fingers are touching the muscle with minimum or no pressure.

In the coordinate system shown in Figure 1, the pull action dynamics can be described by the linear and rotational equations of motion below:

$$
\begin{aligned}
& x=v t+x_{o} \\
& \theta_{t}=\omega t+\theta_{o}
\end{aligned}
$$

where $v$ is the linear velocity of displacement, $\omega$ is the angular velocity of rotation and $x_{o}, \theta_{o}$ are initial coordinates for the displacement and angle of rotation.

\subsection{The Press Action}

The press action is the reverse of the pull action. The motion begins with the tips of the fingers touching the muscle, as in Figure 1.c, and as the masseur/masseuse presses forward, the fingers and the palm end with complete contact and pressure on the muscle, as shown in Figure 1.a; therefore, the press action dynamics are described by the reverse of eqns. 1 and 2 as follows:

$$
\begin{gathered}
x=-v t+x_{2} \\
\theta_{t}=-\omega t+\theta_{\max }
\end{gathered}
$$

For the press action, the initial angle of touch is denoted as $\theta_{t i}=\theta_{\max }$, and the final angle is $\theta_{t f}=0$. On the other hand, the linear displacement for the press action starts at 
(a)

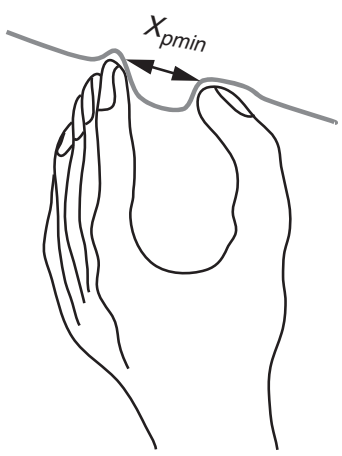

(b)

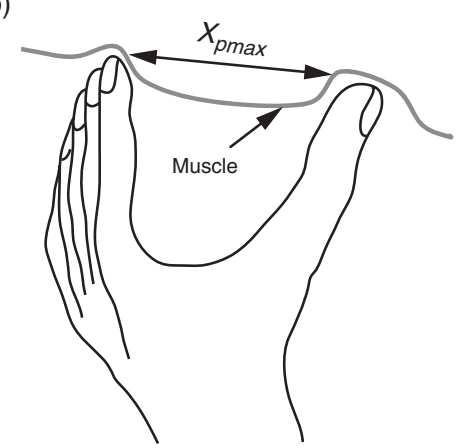

Figure 2. Pin motion. Pin action using fingers and thumb to repeatedly pin the muscle from $x_{p \min }$ to $x_{p \max }$.

the location where the pull action ended; i.e., $x_{i}=x_{2}$ (Figure 1.c) and ends at $x=0$ (Figure 1.a). The negative signs in eqns. 3 and 4 indicate that the motion is opposite to the pull action and the linear and angular velocities are reversed.

\subsection{The Pin Action}

The pin action is performed at the end of the press action using the thumb and fingers. While the muscle is pressed by the hand, the pin action starts with the thumb and the other four fingers coming together, pressing the muscle with a claw action, followed by a release. The pin action can repeat on the same location (see Figure 2) or begin at different locations as the hand moves. Since the closing and opening of the fingers are limited by the size of the hand and the grip on the muscle, the thumb and fingers can close to a minimum pin distance, denoted by $x_{p m i n}$, and open to a maximum pin distance of $x_{\text {pmax }}$.

The cyclic motion of the pin action can be best described by an equation in the following form:

$$
x_{p}=x_{\text {pmax }} \sin (\omega t+\varphi)+c
$$

where $x_{p}$ is the pin displacement of the fingers, $x_{p \max }$ is the maximum opening of the fingers, $\omega$ is the frequency of the motion, $\varphi$ is the phase angle that starts the motion at $x_{p \min }$, and $c$ is a constant.

\subsection{Development of the Mechanical Hand for Thai Massage}

The $\mathrm{MH}$ for Thai massage has to at least be able to reproduce, in an approximate manner, the three $\mathrm{P}$ actions, i.e., pull, press and pin, as described above. Figure 3 shows a conceptual model of the MH. The MH must have a linear motion on the $x y$-plane and rotate in both $z x$ - and $z y$-planes. Only the $z x$-plane rotation is shown in Figure 3.a. For clarification, the $x y$-plane is defined by the frame of the backrest of a chair and the 
(a)

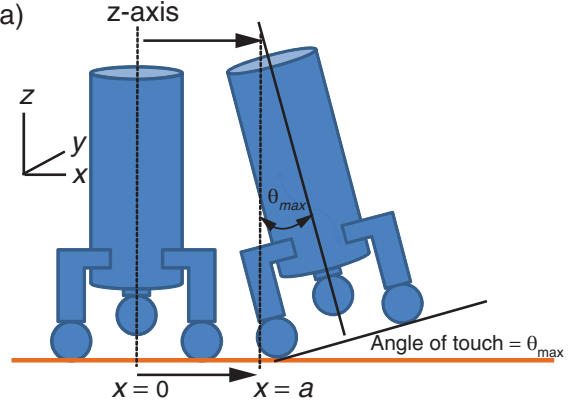

(b)

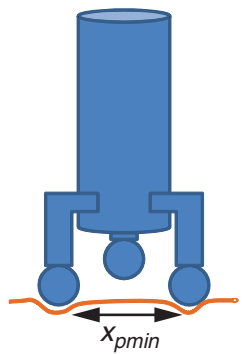

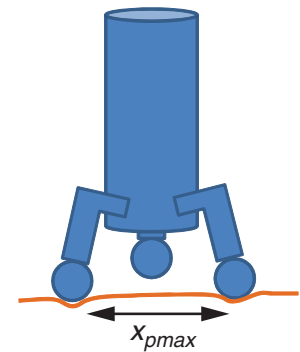

Figure 3. Model of the mechanical hand for the pull, press, and pin actions of Thai massage. (a) The hand rotates on the $z x$-plane and translates on the $x y$ plane. (b) The mechanical hand features finger-like components that open and close to perform the pin action. The angle of touch is defined as the rotation angle of the mechanical hand.

positive $z$-axis is perpendicular to the backrest plane towards the back of the user. To perform the pin action, the mechanical hand must have finger-like components that close and open, as shown in Figure 3.b.

\subsection{Motion on the $x y$-Plane.}

In order to accomplish linear motion on the $x y$-plane, the $\mathrm{MH}$ is mounted on a carriage attached to threaded shafts driven by electric motors, denoted as $M_{x}$ and $M_{y}$ for the linear motion in the $x$ and $y$ directions respectively. The $x y$-displacement occurs in the up and down direction and sideways of the backrest (see Figure 8). Each motor rotates with an angular frequency of $\omega_{x}$ or $\omega_{y}$, respectively. In general, the rotational motion of a threaded shaft converts to the linear motion of a nut on the shaft by the relation $v=\frac{d \omega}{2 \pi}$, where $v$ is the linear velocity, $d$ is the lead of the shaft in $\mathrm{cm} / \mathrm{rev}$ and $\omega$ is the number of revolutions per unit time of the motor $M$ driving the shaft. Equation 1 provides the linear displacement of the carriage in $x$ and $y$ directions given by the following:

$$
\begin{aligned}
& x=v_{x} t+x_{o}=\frac{d \omega_{x}}{2 \pi} t+x_{o} \\
& y=v_{y} t+y_{o}=\frac{d \omega_{y}}{2 \pi} t+y_{o},
\end{aligned}
$$

respectively.

\subsection{Rotational Motion on the $z x$ and $z y$ Planes}

In order to decrease pressure on the muscle and create the pull action, as described in Figure 1, the MH must rotate as shown in Figure 3.a. The rotation increases the angle of touch $\theta_{t}$ from zero to a certain maximum angle, $\theta_{t}=\theta_{\max }$. The mechanics for creating 
(a)

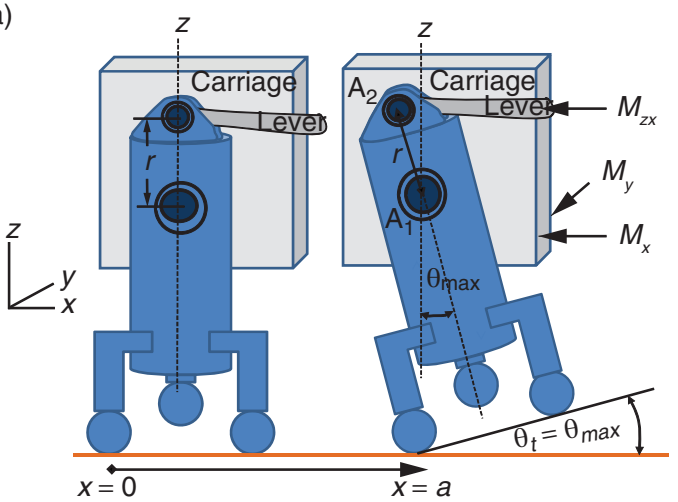

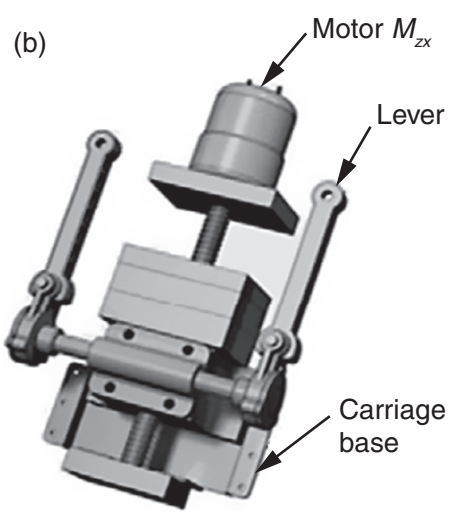

Figure 4. Mechanical hand rotation on the $z x$-plane. (a) The mechanical hand is attached to the carriage by axle $A_{l}$. The lever is attached to the MH at $A_{2}$. Motors $M_{x}$ and $M_{y}$ move the carriage on the $x y$-plane. Motor $M_{z x}$, mounted on the carriage, moves the lever for hand rotation on the $z x$-plane. $r$ is the distance from the center of $A_{1}$ to the center of $A_{2}$. (b) Actual rendering of levers and motor $M_{z x}$.

this motion is shown in Figure 4. The mechanical hand is attached to the carriage at axle $A_{1}$, and a lever attached to the $\mathrm{MH}$ at axle $A_{2}$ is linearly driven by motor $M_{z x}$, pushing the base of the $\mathrm{MH}$ to increase the angle of touch or reversely to decrease it. Therefore, $\theta_{t}$ is related to the linear motion generated by motor $M_{z x}$. Equation 2 shows that the angular velocity of the $\mathrm{MH}$ rotation is related to the linear velocity of the lever, which in turns is generated by the rotation of $M_{z x}$, hence the following:

$$
\theta_{t}=\omega t=v t / r=\frac{d \omega_{z x}}{2 \pi r} t
$$

where $\omega_{z x}$ is the angular frequency of the motor $M_{z x}$ and $r$ is the distance from the center of the hand rotation to the lever attached to $M_{z x}$. $\theta_{t}$ decreases to zero by simply changing the direction of the rotation of $M_{z x}$.

Equation 8 shows that for a constant $\omega_{z x}, \theta_{\max }$ depends on the time $t$ the motor $M_{\mathrm{zx}}$ is activated; thus, we can program the activation of $M_{\mathrm{zx}}$ for $t=t_{1}, t_{2}$, etc., corresponding to different values of $\theta_{\max }$, with the maximum being $60^{\circ}$.

The motion of the $\mathrm{MH}$ on the $z y$-plane is accomplished by a tapered camshaft attached to location $A_{l}$ by ball bearings (see Figure 5). The base of the $\mathrm{MH}$ is held by the lever arm on the $x$-axis; when the tapered camshaft rotates, the MH rotates back and forth on the $z y$-plane, sweeping an angle with the pivot point at $A_{1}$. To describe the dynamics on this plane, we denote the tapered angle of the camshaft as $\theta_{o}$ (see Figure 5.b); as the camshaft rotates, it pulls the mechanical hand in and out on the zy-plane and rotates the $\mathrm{MH}$ on the $x y$-plane from $-\theta_{o}$ to $+\theta_{o}$, generating a twisting motion on this plane and thus enhancing the massage experience. 
(a)

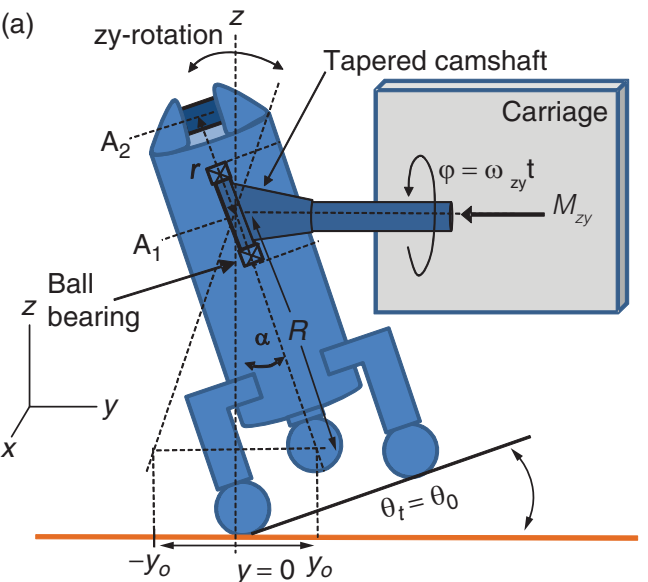

(b)

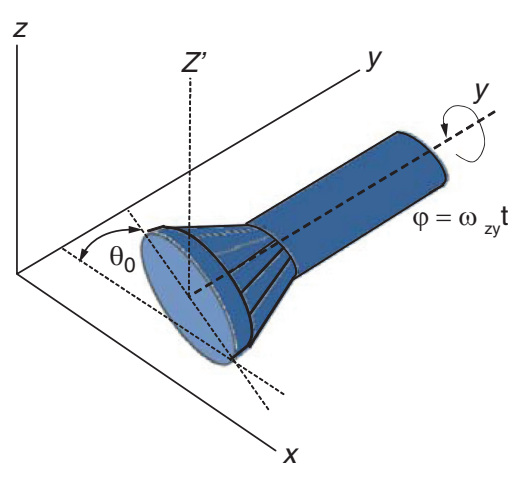

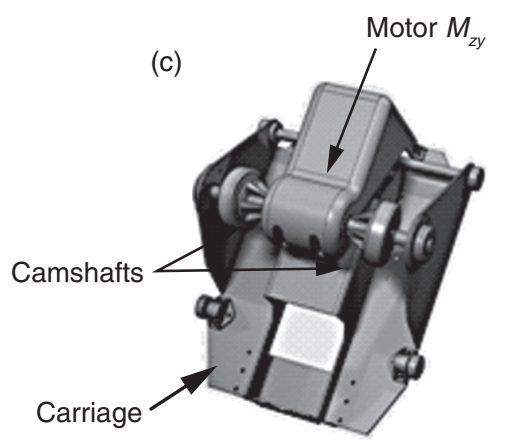

Figure 5. Mechanical hand rotation on the $z y$-plane. (a) The mechanical hand is attached to the carriage by axle $A_{l}$ with a tapered camshaft. The lever arm attached to $A_{2}$ holds the $\mathrm{MH}$ on the $z x$-plane while the rotation of the camshaft by motor $M_{\mathrm{zy}}$ generates the rotation of the $\mathrm{MH}$ on the $z y$-plane. (b) Camshaft at its initial location at $t=0$. At this location, the tapered angle $\theta_{\mathrm{o}}$ lies on the $x y$-plane with zero projection on the $z y$-plane; as the camshaft rotates $90^{\circ}$ clockwise, the projection angle a is on the $z y$-plane, causing the $\mathrm{MH}$ to rotate on the $z y$-plane, as shown in (a). (c) Actual rendering of camshafts mounted on the carriage.

Let $\alpha$ be the projection of $\theta_{o}$ onto the $z y$-plane and note that $\alpha$ oscillates from $\theta_{o}$ to $-\theta_{o}$. Figure 5 .a shows that the displacement of the center finger of the hand along the $y$-axis is given by

$$
\mathrm{y}=R \sin (\alpha)
$$

where $y_{o}=R \sin \left(\theta_{o}\right)$, the maximum displacement in the $y$-axis due to the rotation of the camshaft, and $R$ is the distance from $A_{l}$ to the center of the middle finger. Now let $\varphi$ be the angle of rotation of the camshaft and position the camshaft so that at $t=0, \varphi=0$. 
At this location (see Figure 5.b) the projection of the tapered angle of the camshaft onto the $x y$-plane is $\theta_{o}$, but there is no projection onto the $z y$-plane; i.e., the face of the camshaft lies parallel to the $z$-axis, as shown in Figure 5.b. As $\varphi$ increases from zero to $\pi / 2$, the projection angle $\alpha$ of $\theta_{o}$ on the $z y$-plane increases from zero to $\theta_{o}$ (this is the case depicted in Figure 5.a). At this location, the face of the camshaft makes an angle with the $z$-axis on the $z y$-plane; for $\varphi=\pi, \alpha$ is back to zero; for $\varphi=3 \pi / 2, \alpha=-\theta_{o}$; and for $\varphi=2 \pi, \alpha=0$. Therefore, $\alpha$ is related to the rotation angle $\varphi$ by the following equation:

$$
\alpha=\theta_{o} \sin \varphi
$$

with $\varphi=\omega_{z y} t$, where $\omega_{z y}$ is the angular frequency of the motor $M_{z y}$ driving the camshaft, and thus the time dependence of $\alpha$ is given by

$$
\alpha=\theta_{o} \sin \left(\omega_{z y} t\right)
$$

Using eqns. 10 and 11, the dynamics of the pull and press actions on the $z y$-plane is given by:

$$
\mathrm{y}=R \sin (\alpha)=R \sin \left(\theta_{o} \sin \left(\omega_{z y} t\right)\right)
$$

Adding the displacement of the carriage along the $y$-axis, the total displacement of the center finger of the $\mathrm{MH}$ is given by the following equation:

$$
\mathrm{y}=R \sin \left(\theta_{\mathrm{o}} \sin \left(\omega_{z y} t\right)\right)+\frac{d \omega_{y}}{2 \pi} t+b_{o},
$$

where $b_{o}$ is the initial location of the carriage in the $y$-direction at $t=0$. On this plane, the angle of touch $\theta_{t}=\alpha$. Equations 11 and 13 give the angle of touch and location of the $\mathrm{MH}$ in the $y$-direction. The maximum pressure occurs when all fingers are on the muscle, i.e., when $\alpha=0$, and as the camshaft rotates, the muscle is pulled and pressed to the left and to the right in the $y$-direction. Thus, together with the $x$-axis pull and press actions, the mechanical hand provides a comprehensive massage to the muscle.

\subsection{The Pin Action}

In addition to the press and pull actions, a pin action needs to be performed. For this action, the mechanical fingers of the hand must open and close, as shown in Figure 3.b. The mechanical hand has four fingers (see Figure 6.a), three movable and one centered for stability and evenness during the pull and press actions of the massage. The pin motion is accomplished using a sinusoidal surface flywheel or camshaft that pushes on the levers attached to the mechanical fingers, as shown in Figure 6.c.

The temporal motions of the fingers are generated by the finger lever through rotation of the sinusoidal camshaft. At $t=0$, the levers are at a minima and as the camshaft begins to rotate, the levers reach a maximum downward displacement at $\pi / 3$ 
(a)

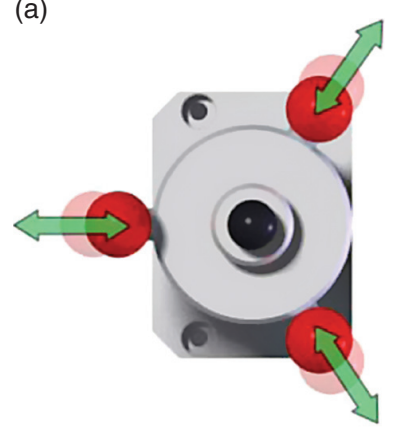

(b)

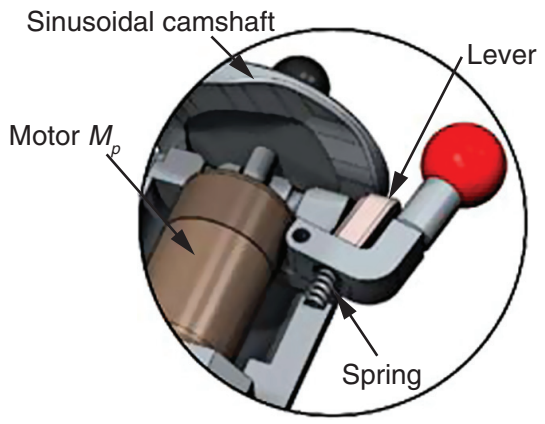

(c)

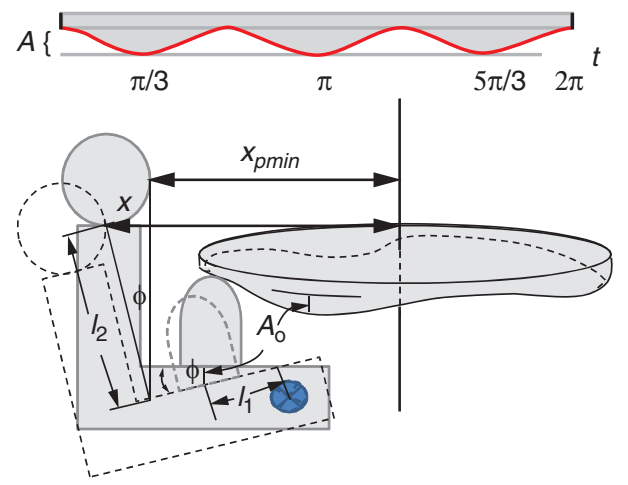

Figure 6. Motion of the mechanical fingers. (a) The MH has four fingers: one central and three peripheral; the peripheral fingers move outwards relative to the fixed central finger. (b) Actual design of a mechanical finger showing the sinusoidal camshaft pressing the lever that opens and closes the fingers. (c) Side view of the camshaft extended in two dimensions; the maximum downward motion of the finger lever to a maximum amplitude $A_{o}$ occurs at angles of rotation equal to $\pi / 3, \pi, 5 \pi / 3$. As the amplitude of the camshaft increases, it pushes the finger lever downward a distance $A$, moving the finger arm down by an angle $\phi$, opening the finger an extra distance $x ; l_{1}$ and $l_{2}$ are the measured distances.

radians of rotation, a minimum at $2 \pi / 3$, a maximum at $\pi$, and so forth until it completes one revolution. This downward motion of the levers can be described using a sinusoidal equation of the form:

$$
D(t)=\frac{A_{o}}{2}\left[\sin \left(\omega_{p} t+\varphi_{p h}\right)+1\right]
$$

where $D(t)$ is the temporal displacement of the levers, $A_{0}$ is the maximum height or amplitude of the sinusoidal camshaft, $\omega_{p}$ is the angular frequency of the camshaft generated 
by the pin motor $M_{\mathrm{p}}, \varphi_{p h}$ is the phase angle that starts the levers at a minimum or at a maximum. Hence, the phase angle for the initial minima is given by setting $D(0)=0$ :

$$
D(0)=\frac{A_{o}}{2}\left[\sin \left(\omega_{p} 0+\varphi_{p h}\right)+1\right]=0 \text { or } \varphi_{p h}=-\pi / 2 .
$$

We also can select $\omega_{p}$ so that after a time $t=\pi / 3, D(t)$ is at a maximum. The condition for the frequency then has to satisfy the following relation:

$$
\omega_{p} t+\varphi=\omega_{p} t-\frac{\pi}{2}=\frac{\pi}{2}
$$

therefore, for $t=\pi / 3, \omega_{p}=3$. Figure 6.c shows a representation of the sine function as a function of time, matching the geometric form of the disk. From Figure 6.c we can see that the temporal opening and closing of the mechanical fingers are related to the vertical displacement $D(t)$ of the levers through the rotation angle $\phi$ :

$$
\sin \phi=\frac{D(t)}{l_{1}}=\frac{x(t)}{l_{2}} \quad \text { or } \quad x(t)=\frac{l_{2}}{l_{1}} D(t)=L D(t),
$$

where $L=\frac{l_{2}}{l_{1}}, l_{1}$ and $l_{2}$ are measured distances on the finger arms, as shown in Figure 6.c.

The temporal opening and closing of the fingers then can be written as:

$$
X(t)=x(t)+x_{p \min }=L D(t)+x_{p \min }
$$

Using eqn. 14,

$$
X(t)=L \frac{A_{o}}{2}\left[\sin \left(\omega_{p} t+\varphi_{p h}\right)+1\right]+x_{p \min }
$$

Equation 19 describes the temporal motion of the mechanical fingers.

\subsection{Temporal Dynamics and Programming of the Motion of the 3 P's}

Figure 7 shows the actual representation of the mechanical hands mounted on the carriage. The " $3 \mathrm{P}$ actions" of pull, press, and pin are carried out by activating the motors $M_{x}, M_{y}, M_{z x}, M_{z y}$ and $M_{p}$, at predetermined times. For instance, the synchronized activation of $M_{x}$ and $M_{z x}$ generates the pull action along the $x$-axis, and the reverse generates the press action. On the other hand, activation of $M_{x}$ and $M_{p}$ generates the pin action up or down a muscle in the $x$-direction. Equations $6,7,8,14$ and 19 describe the mechanical motion of the hand as a function of time, but mechanical motion occurs only if motors $M_{x}, M_{y}, M_{z x}, M_{z y}$ and $M_{p}$ are activated at predetermine times. 
(a)

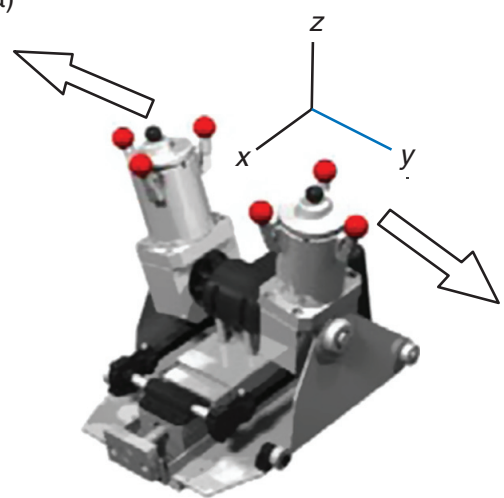

(b)

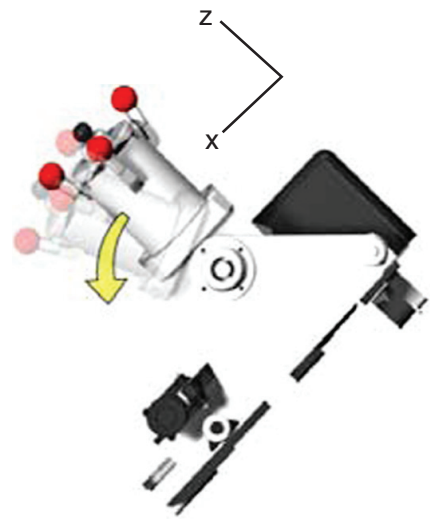

Figure 7. Mechanical hands mounted on the carriage. (a) Motion of the MH on the $z y$-plane. (b) Motion of the MH on the $z x$-plane.

(a)

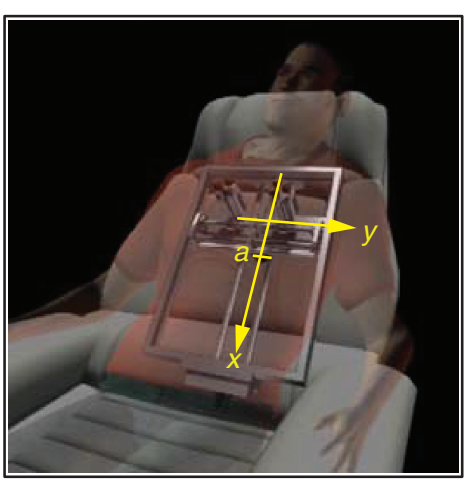

(b)

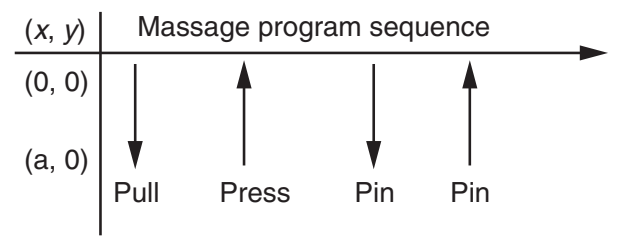

Figure 8. Mechanical hands positioned on the backrest. (a) The mechanical hands mounted on the carriage and positioned in an arbitrary location designated as the initial starting point $(x, y)=(0,0)$. (b) A typical massage program starts at $(0,0)$, pull to $(a, 0)$, press from $(a, 0)$ to $(0,0)$, and followed by pinning from $(0,0)$ to $(\mathrm{a}, 0)$ and back to $(0,0)$.

By using an electronic controller, we can easily program the motors $M_{x}, M_{y}, M_{z x}, M_{z y}$ and $M_{p}$ to generate the 3 P's of Thai massage. We have opted for the use of Unitronics ${ }^{\circledR}$ Vision 120 controller for the time activation of the motors. Unitronics ${ }^{\circledR}$ 's VisiLogic Ladder Programming (UVLP) is easy to use and is appropriate for this type of mechanical system. Step-by-step procedures are found in the UVLP manual given in reference [17]. The program sequencing required for the activation of the motors will be described.

For a massage sequence shown in Figure 8.b, the temporal activation of the motors for the pull action is accomplished by positioning the carriage at $(0,0)$, then moving 


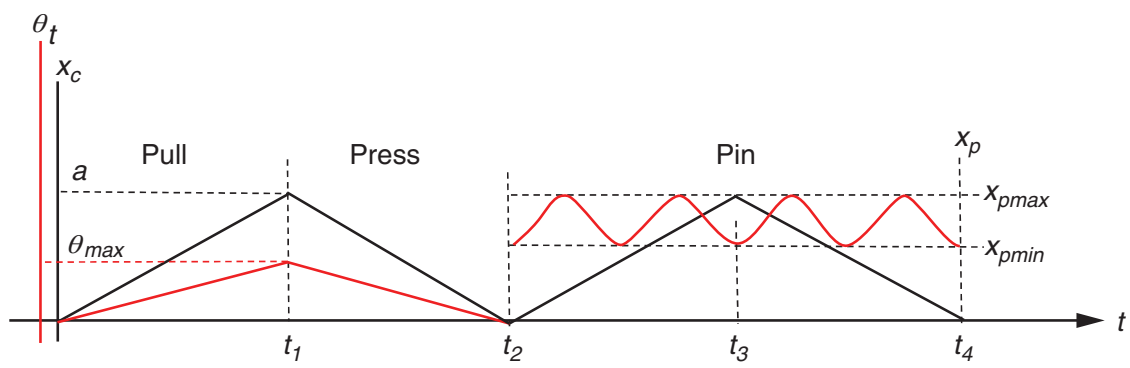

Figure 9. Temporal representation of the pull, press, and pin sequence described in Figure 8 . The three vertical axes indicate the displacement of the carriage along the $x$-axis, the increase of the angle of touch $\theta_{t}$, and the opening and closing of the mechanical fingers.

distance $x=a$. This motion is performed by the activation of $M_{x}$ for time $t=t_{1}$ described by eqn. 6 . The pressure on the muscle is decreased by generating the rotational motion of the $\mathrm{MH}$, as shown in Figure 2. This rotational motion is accomplished by activating $M_{z x}$ while motors $M_{y}, M_{z y}$ and $M_{p}$ are turned off. The angular velocity of the rotation is such that during the same time $t=t_{1}$, the rotation reaches the maximum angle of touch $\theta_{t}=\theta_{\max }$. A graphical representation of the motion is presented in Figure 9. For both the press and pull actions, the displacement in $x$ (eqn. 6) and the change in angle $\theta$ (eqn. 8) are linear in time of the form $x=m t+a$ and $\theta_{t}=m t+\theta_{o}$; on the other hand, for the pin action the displacement of the fingers is sinusoidal, as given in eqn. 19.

In Figure 9, from $t_{1}$ to $t_{2}$, the motions of both the carriage and the rotation of the $\mathrm{MH}$ are reversed to press the muscle until the carriage is back to $x=0$ and $\theta_{t}=0$ for maximum pressure (black and red lines from $0=>t_{1}=>t_{2}$, Figure 9). From $t_{2}$ to $t_{3}$, the carriage moves to $x=a$ with a simultaneous pin action by the mechanical fingers, and finally from $t_{3}$ to $t_{4}$, the carriage moves back to $x=0$ with continued pin action. For this particular sequence, only motors $M_{x}, M_{z x}$ and $M_{p}$ are activated.

The previous massage sequence is repeated several times to warm up the muscle. After the warm up period, a more complex massage sequence is initiated. For instance, the carriage is displaced down the $x$-axis with simultaneous pull and press actions in the $y$-direction, including pin action simultaneously. This massage sequence is depicted in Figure 10.

In this massage sequence, motors $M_{x}, M_{z y}$ and $M_{p}$ are activated. The carriage moves from $x=0$ to $a$ back to 0 as given in eqn. 6 , while the pull and press actions in the $y$-axis is given in eqn. 11, and the pin action is given in eqn. 19. The massage sequences described in Figures 9 and 10 show the flexibility of our mechanical system. By appropriately activating motors $M_{x}, M_{y}, M_{z x}, M_{z y}$ and $M_{p}$, we can program various massage sequences for the upper, medium, and lower back, with vertical and transverse pull and press actions, with or without pin action.

A typical massage sequence takes around 7.5 seconds to complete a cycle of the pull, press, and pin actions per massage area. Of the 7.5 seconds, it takes about 3 seconds for the pull action, 3 seconds for the press action, and 1.5 seconds for the pin. The massage 


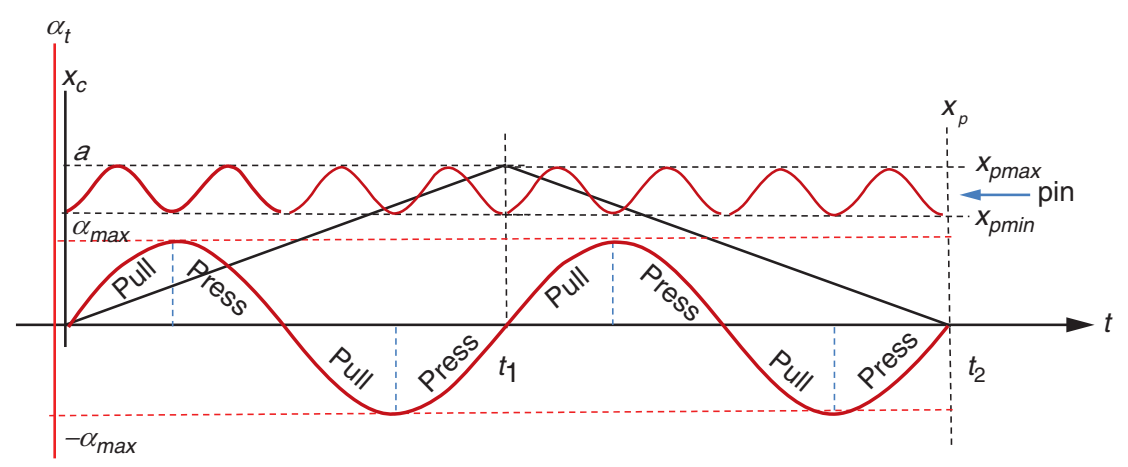

Figure 10. Temporal representation of a complex massage sequence. Massage sequence with the motion of the carriage along the $x$-axis, rotation of the mechanical hand with respect to the $y$-axis, and pin action simultaneously. From $t=0$ to $t_{1}$, the carriage is displaced linearly from 0 to $x=a$; during this time, the $\mathrm{MH}$ rotates per eqn. 11 pulling and pressing in both directions of the $y$-axis, with simultaneous pin action. From $t_{1}$ to $t_{2}$ the carriage returns to $x=0$ while the pull, press, and pin actions are repeated.

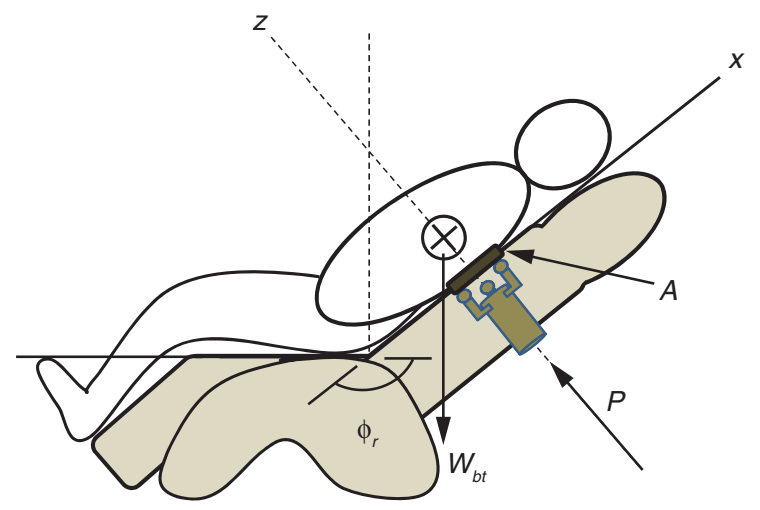

Figure 11. Geometry for the estimation of massage pressure. The pressure $P$ (see eqn. 20) on the back of the user depends on the reclining angle $\phi_{r}$, the component of the body trunk weight $W_{b t}$ perpendicular to the backrest along the $z$-axis, the area $A$ covered by the MH fingers, and the angle of touch $\theta_{\mathrm{t}}$, defined in Figure 1 .

sequence repeats twice on the same area for a total of 15 seconds before moving to the next area. When the hand mechanism moves upward, downward, or sideways to another area, the pull action will take around 6 seconds depending on the program sequence selected.

\subsection{Prototype Testing}

This research was reviewed and approved by the Review Board of the Integrated Product Design and Manufacturing Program, School of Energy, Environment, and 
(a)

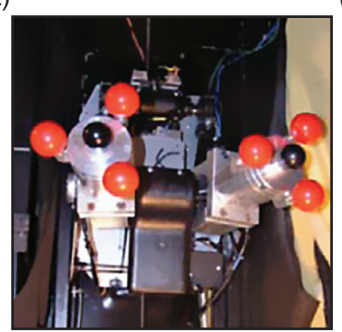

(b)

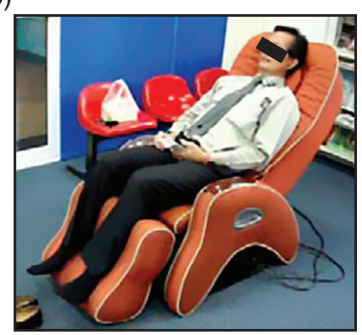

(c)

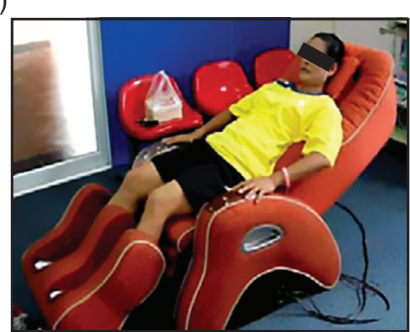

Figure 12. Experimental setup. (a) Prototype of the Mechanical Massaging System installed in the backrest of the chair. (b) and (c) Prototypes of the massage chair tested by participants.

Materials, King Mongkut's University of Technology Thonburi, in Thailand. It was also approved by the Ethics Committee of the same institution. The participants of the Ministry of Tourism and Sports consented to the tests by signing a test form.

We mounted the MH set on the backrest of a prototype chair (see Figure 12) and tested its functionality and user satisfaction. The participants group for this initial test consisted of 20 males and 20 females recruited from the city of Bangkok. Individuals with medical back conditions were excluded. Before participating in the trial, each of the participants signed a consent form to indicate that he/she was fully informed of any risks involved. Each participant tested the MH Thai massage (Figure 12b) for a period of 10 minutes with pull, press, and pin sequences from the upper to the lower back (along the $x$-axis, as shown in Figure 9), as well as pull, press, and pin sequences sideways (in the $y$-direction) and down (as shown in Figure 10).

The backrest of this prototype has dimensions of $33 \mathrm{~cm} \times 60 \mathrm{~cm}$, which accommodates small- to medium-size individuals 130 - to $190-\mathrm{cm}$ tall. Tall men in Thailand average $169.46 \mathrm{~cm}$ in height, while women average $157 \mathrm{~cm}$. Taller individuals with longer and wider backs, such as Europeans and Americans, can be accommodated by a larger system with an MH separation along the $y$-axis appropriate for their sizes.

In this massage system, the massage pressure depends on the reclining angle, the weight of the person, and on the angle of touch. The pressure on the area covered by the $\mathrm{MH}$ is calculated by using the component of the trunk weight perpendicular to the area covered by the MH's:

$$
P=\frac{W_{b t}}{2 A} \sin \left(\phi_{r}-90\right) \cos \theta_{t}
$$

where $P$ is the pressure, $W_{b t}$ is the body trunk weight, $A$ is the area covered by the $\mathrm{MH}$, $\phi_{r}$ is the reclining angle, and $\theta_{t}$ is the angle of touch (see Figure 11).

From eqn. 20, we can see that for any reclining angle, the maximum pressure occurs when the angle of touch is zero and the area covered by the $\mathrm{MH}$ is a minimum; i.e., the mechanical fingers are closed or at $38.48 \mathrm{~cm}^{2}$. The body trunk weight corresponds to $50.8 \%$ of the body weight [19]. The mean mass of the sample group was $68.2 \mathrm{~kg}$ for men and $57.9 \mathrm{~kg}$ for women. Thus, for a reclining angle of 130 degrees, the massage pressure for men was light (64\% of the body trunk weight) or $28.91 \mathrm{kPa}$, for 145 
degrees or medium pressure (81.9\% of the body trunk weight) it was $37 \mathrm{kPa}$, and for 160 degrees or high pressure (94\% of the body trunk weight) it was $42.46 \mathrm{kPa}$. The corresponding values for women were $24.54,31.41$ and $36.04 \mathrm{kPa}$, respectively.

In TTM, the pressure depends on the weight of the masseur/masseuse and the inclination angles that the masseur/masseuse uses over the subject. If the masseuse is a typical Thai woman with an average mass of $57.9 \mathrm{~kg}$, a maximum inclination angle of 60 degrees from the vertical, and a hand area of $98 \mathrm{~cm}^{2}$, the pressure is of the order of $25.58 \mathrm{kPa}$. For a man, the pressure would be $30.13 \mathrm{kPa}$. The advantage of our system over the TTM in regards to pressure is the ability of the user to select a comfortable massage pressure that can be increased gradually over time.

The participants were asked to rate on a scale from 1 to 5 the performance of the mechanical-hand massage based on two key questions:

(a) To what extent does the Thai massage by the MH perform the pull, press, and pin actions like a human masseur/masseuse?

(b) What is your overall satisfaction with the Thai massage by the current massage system?

\section{RESULTS}

The subjects answering question (a), on a scale from 1 to 5 , gave an average score of 3.51 with a standard deviation of 0.99 ; i.e., the subjects felt that the massage was close to a Thai massage performed by human hands. They felt the pull, press, and pin actions were well defined and easy to distinguish. When we analyzed the responses by gender, we found that males gave a higher score, 3.57, while the females gave 3.44. As presented in Table 1. Thirty percent of the participants, both men and women, stated that it needed more grabbing of the muscle in the pin action. This is understandable and will be improved in the future system. In our model, the mechanical fingers start at $x_{\text {min }}$ $=35 \mathrm{~mm}$ from the central axis of the $\mathrm{MH}$ to a maximum opening distance of $53.5 \mathrm{~mm}$, a displacement of $18.5 \mathrm{~mm}$. The padding and fabric material between the mechanical fingers and the skin of the user reduces the pin action effect considerably. Taking into consideration the thickness of the padding, the mechanical fingers need to oscillate between $x_{\min }=25 \mathrm{~mm}$ and $x_{\max }=55 \mathrm{~mm}$, a span of $30 \mathrm{~mm}$. This adjustment will be implemented in the next version of the $\mathrm{MH}$.

In regards to the massage pressure, most users found that reclining angles from $112-135$ degrees were very comfortable (64\% of the upper body weight); however, some users preferred a heavier massage, and adjusted the reclining angle between 145 and the maximum at 170 degrees. In this range, the users that were allowed to test for more than 15 minutes stated that they began to feel muscle strain. In general, it is advised that mechanical massage systems be used for no more than 15 minutes.

Table 1. Results for question (a) response.

\begin{tabular}{|c|c|c|c|c|c|c|c|c|c|c|c|c|c|c|c|c|c|c|c|c|c|}
\hline Subject & 1 & 2 & 3 & 4 & 5 & 6 & 7 & 8 & 9 & 10 & 11 & 12 & 13 & 14 & 15 & 16 & 17 & 18 & 19 & 20 & Mean \\
\hline Male & 4 & 4 & 4 & 4 & 4 & 5 & 3 & 0 & 4 & 3 & 2 & 4 & 3 & 4 & 4 & 3 & 4 & 3 & 3 & 3 & 3.57 \\
\hline Female & 3 & 4 & 0 & 3 & 4 & 3 & 4 & 4 & 3 & 4 & 4 & 3 & 4 & 3 & 3 & 4 & 3 & 4 & 0 & 3 & 3.44 \\
\hline
\end{tabular}


Table 2. Results for question (b) response.

\begin{tabular}{|c|c|c|c|c|c|c|c|c|c|c|c|c|c|c|c|c|c|c|c|c|}
\hline Subject & 1 & 2 & 3 & 4 & 5 & 6 & 7 & 8 & 9 & 10 & 11 & 12 & 13 & 14 & 15 & 16 & 17 & 18 & 1920 & Mean \\
\hline Male & 4 & 3 & 3 & 5 & 3 & 4 & 3 & 5 & 3 & 4 & 3 & 5 & 3 & 4 & 4 & 3 & 0 & 3 & 43 & 3.63 \\
\hline Female & 3 & 4 & 0 & 3 & 4 & $y$ & 4 & r & $J$ & 4 & 4 & 3 & 4 & 3 & 3 & 4 & 3 & 4 & 03 & 3.55 \\
\hline
\end{tabular}

As for question (b), regarding the participants' satisfaction with the MH chair Thai massage system, the satisfaction score was 3.59 with a standard deviation of 0.76 . With respect to gender, it was found that males were more satisfied than females, with a satisfaction score of 3.63 and 3.55, respectively, as presented in Table 2. Further, $72 \%$ of the participants felt that the MH performed more like a human hand massage than other massage mechanical systems they had used.

The participants were also interviewed after the test and were asked for suggestions to improve the current $\mathrm{MH}$ chair system. Several improvements were suggested, including softer or bigger mechanical fingers or a thicker cushion, additional leg massage, and leather covering instead of fabric. Seventy percent of the participants thought that the MH performed satisfactorily overall.

\section{DISCUSSION}

The MH for Thai massage is the first of its kind. It provides the basis for more advanced and complex robotic systems for relaxation massage and possibly medical massage treatments. Its rotational and translational motion, with mechanical fingers to generate the pull, press and pin, is the first attempt to mechanically produce a basic and standard form of Thai massage. This in itself is a limitation of this work because, as we mentioned in the introduction, Thai massage is very comprehensive and has many variations to the extent of being an art form of massage, which needs to be studied more scientifically. Nevertheless, we were able to identify the 3 P's, pull, press, pin, as the key characteristic actions of royal Thai massage. Based on these characteristic actions, we prototyped a mechanical system to deliver the massage. Future research should study different types of Thai massage in more detail so that a design can be developed specifically for each of the types.

Another limitation of this work involved the performance testing of the prototype massage chair. The participants were asked simple questions and they gave positive responses. We are not sure, however, whether the participants really liked the system and would actually acquire a Thai massage system once it is on the market. Further tests with focus groups as well as with individuals with physical therapy needs for the back, and more specific data collection techniques, can yield more insight into the medical market potential of this system. Moreover, research using medical equipment such as MRI scans could provide a more objective perspective on the therapeutic effects that the test participants experience.

In addition, more research must be done with larger sample sizes, including patients with chronic back pain, and using an MH system with more human-like fingers, i.e., bigger and softer fingers, keeping in mind that the size of the mechanical parts must fit within the backrest of the chair. 
On the other hand, the system can present a solution for standard back pain therapy with controlled pressure, controlled massage sequences, and consistent massage techniques. Obviously, the system cannot completely duplicate actual hand massage, but it can deliver simulated basic hand motions required for the three Ps in a controlled manner. This offers an advantage over hand massage because the user can systematically increase or decrease the massage intensity in terms of speed and pressure, change the location, and start/terminate the massage anytime as desired. Normally, mechanical massage systems should not be used for more than 15 minutes per session, especially at a high intensity. Massages exceeding this time may strain the muscles and cause pain. A lower intensity massage can be achieved, if desired, by simply reducing the reclining angle of the system, or selecting a smaller angle of touch of the $\mathrm{MH}$.

\section{CONCLUSION}

We have described and developed a set of mechanical hands for the standard delivery of traditional Thai massage. A mathematical model and the mechanics of the three main actions of Thai massage, i.e., pull, press, and pin, were developed, and a prototype of the mechanical hands was built and tested. The independent motion of the hand carriage, hand rotation, and the motion of the mechanical fingers allowed for the programming of repetitive complex massage sequences simulating a standard massage.

The prototype of the traditional Thai massage system was tested with forty users, and the results indicated that the prototype can perform massage more "human-like" than that by existing roller-type massage systems, with about seventy percent of the participants considering that the MH performed satisfactorily overall. This initial testing suggested that a mechanical system for the standard delivery of traditional Thai massage to patients in clinics and hospitals is possible, and could be a component of integrated healthcare.

\section{ACKNOWLEDGEMENTS}

The authors would like to acknowledge the following organization and people that made this work possible: the Ministry of Tourism and Sports for providing the test subjects, and Dr. Angel Castellanos for verifying the modeling.

\section{CONFLICT OF INTEREST}

The authors declare that there is no conflict of interest.

\section{NOMENCLATURE}

$\begin{array}{ll}a & \text { Displacement constant, } \mathrm{cm} \\ A & \text { Area covered by the mechanical hand, } \mathrm{cm}^{2} \\ A_{1} & \text { Axle one, dimensionless } \\ A_{2} & \text { Axle two, dimensionless } \\ A_{o} & \text { Maximum height of the sinusoidal camshaft, } \mathrm{cm} \\ b_{o} & \text { Initial location of carriage in the y-axis, } \mathrm{cm} \\ d & \text { Lead of shaft, } \mathrm{cm} / \mathrm{rev} \\ D(t) & \text { Temporal displacement of the mechanical finger levers }, \mathrm{cm} \\ l_{1}, l_{2} & \text { Lengths of mechanical finger arms, } \mathrm{cm}\end{array}$


$L \quad$ Ratio of $l_{2} / l_{1}$, dimensionless

$M_{x} \quad$ Motor for displacement in the $x$-direction, dimensionless

$M_{y} \quad$ Motor for displacement in the $y$-direction, dimensionless

$M_{z x} \quad$ Motor for rotation on the $z x$-plane, dimensionless

$M_{z y} \quad$ Motor for rotation on the $z y$-plane, dimensionless

$M_{p} \quad$ Motor for pin motion, dimensionless

$P \quad$ Pressure, $\mathrm{kPa}$

$r \quad$ Distance between $A_{l}$ and $A_{2}, \mathrm{~cm}$

$R \quad$ Distance between $A_{2}$ and center of the middle mechanical finger, $\mathrm{cm}$

$t \quad$ Time, second

$t_{f} \quad$ Final time, second

$v \quad$ Linear velocity, $\mathrm{cm} / \mathrm{s}$

$W_{b t} \quad$ Body trunk weight, $\mathrm{N}$

$x, x_{2} \quad$ Displacement, $\mathrm{cm}$

$x_{o} \quad$ Initial displacement, $\mathrm{cm}$

$x_{f} \quad$ Final displacement, $\mathrm{cm}$

$x_{p} \quad$ Pin displacement, $\mathrm{cm}$

$x_{\text {pmin }} \quad$ Pin minimum displacement, $\mathrm{cm}$

$x_{\text {pmax }} \quad$ Pin maximum displacement, $\mathrm{cm}$

$X(t) \quad$ Temporal opening distance of the mechanical fingers, $\mathrm{cm}$

Greek

$\alpha \quad$ Angle of touch on the $z y$-plane

$\varphi \quad$ Phase angle for pin motion, radians

$\phi \quad$ Rotation angle of mechanical finger arm, radians

$\phi_{r} \quad$ Reclining angle, degrees

$\theta_{t} \quad$ Angle of touch on the $z x$-plane, degrees

$\theta_{o} \quad$ Initial angle, degrees

$\theta_{\max } \quad$ Maximum angle of touch, degrees

$\omega \quad$ Angular velocity, radians/s

$\omega_{x}, \omega_{y} \quad$ Angular frequency for displacement in the $x$ and $y$ axis respectively, radians/s

$\omega_{z x} \quad$ Angular frequency of $M_{z x}$, radians/s

$\omega_{z y} \quad$ Angular frequency of $M_{z y}$, radians/s

\section{REFERENCES}

[1] Uraiwon Chatchawan, Bandit Thinkhamrop, Sarnerduen Khamwan, Jacqueline Knowles, Wichai Eungpinichpong: Effectiveness of traditional Thai massage versus Swedish massage among patients with back pain associated with myofascial trigger points. Journal of Bodywork and Movement Therapies, 2005, 9(4): 298-309.

[2] Vitsarut Buttagat, Wichai Eungpinichpong, David Kaber, Uraiwon Chatchawan, Preeda Arayawichanon: Acute effects of traditional Thai massage on electroencephalogram in patients with scapulocostal syndrome. Complementary Therapies in Medicine. 2012, 29(4):167-174.

[3] Vitsarut Buttagat, Wichai Eungpinichpong, Uraiwon Chatchawan, Preeda Arayawichanon.: Therapeutic effects of traditional Thai massage on pain, muscle tension and anxiety in patients with scapulocostal syndrome: A randomized single-blinded pilot study. Journal of Bodywork and Movement Therapies. 2012, 16(1): 57-63. 
[4] Vitsarut Buttagat, Wichai Eungpinichpong, Uraiwon Chatchawan, Samerduen Kharmwan: The immediate effects of traditional Thai massage on heart rate variability and stress-related parameters in patients with back pain associated with myofascial trigger points. Journal of Bodywork and Movement Therapies. 2011, 15(1): 15-23.

[5] Lucinda Lidell, Sara Thomas, Carola Beresford Cooke, and Anthony Porter: The book of massage. Gaia Books Limited, London. 1984, 26-27.

[6] Norman J. Marcus, MD, Edward J. Gracely, PhD, and Kelly O. Keefe, A Comprehensive Protocol to Diagnose and Treat Pain of Muscular Origin May Successfully and Reliably Decrease or Eliminate Pain in a Chronic Pain Population, Pain Medicine 2010, 11: 25-34.

[7] Sritoomma Netchanok, Moyle Wendy, Cooke Marie, O’Dwyer Siobhan: The effectiveness of Swedish massage and traditional Thai massage in treating chronic low back pain: A review of the literature. Complementary Therapies in Clinical Practice. 2012, 18(4): 227-234.

[8] Phennapha Sapcharoen, Sombun Kiatinann: The efficacy and satisfaction of clients getting Thai traditional massage at the Institute of Thai Traditional Medicine. Thailand Institute of Traditional Medicine and Alternative Medicine, Ministry of Public Health, Ministry of Public Health. B.E. 2003, 1-4.

[9] Tawee Laohapand, Uapong Jaturatamrong: Thai Traditional Medicine in the Faculty of Medicine Siriraj Hospital, Ayurved Thamrong School. Center of Applied Thai Traditional Medicine, Faculty of Medicine Siriraj Hospital, Mahidol University. Supavanich Press, Bangkok, 2009, 23.

[10] Kanthima Sitthithankit, Phonthip Toemwiset: Guide of Thai Traditional Medicine for Public Health, Thailand Department of Developmental and Alternative Medicine, Ministry of Public Health, The War Veterans Organization of Thailand, Bangkok, 2004, 111-113.

[11] Phennapha Sapcharoen, Kanchana Tiwiset: Museum and Traditional Medicine Training Center Thailand, The Institute of Thai Traditional Medicine. Thailand Department of Developmental and Alternative Medicine. Ministry of Public Health. The War Veterans Organization of Thailand, Bangkok. 2003, 106-108.

[12] Pongjan Yoopat, Christophe Maes, Stefaan Poriau, Kamiel Vanwonterghem. Thai tradition massage: Efficiency-assessment of three traditional massage method on office workers: An explorative study. Journal of Bodywork and Movement Therapies. 2014, 1-7.

[13] Sunee Saetung, La-or Chailurkit, and Boonsong Ongphiphadhanakul: Thai traditional massage increases biochemical markers of bone formation in postmenopausal women: a randomized crossover trial. BMC Complementary \& Alternative Medicine. 2013, 13-69.

[14] Tawee Laohapand, Uapong Jaturatamrong: Thai Traditional Medicine in the Faculty of Medicine Siriraj Hospital. Ayurved Thamrong School, Center of Applied Thai Traditional Medicine, Faculty of Medicine Siriraj Hospital, Mahidol University, Supavanich Press, Bangkok, 2014.

[15] Salinee Rattanaphan and Panya Srichandr, Engineering the human touch: mimicking the art of Thai massage. International Journal of Design and Manufacturing Technology. 2012, 3, 1, 34.

[16] Medical Rehabilitation Foundation promotes Thailand's original Ayurvedic School: Thailand Traditional Therapeutic Massage (Massage Courts). Ganesha FDK Center Co., Ltd., Bangkok, 2005, 23.

[17] Phennapha Sapcharoen, Aphichat Limtiyayothin, Ubolratana Manosin: Royal Thai Massage Training Manual. Thai Traditional Medicine Development Foundation, Thailand. Sam Cha Roen Panich Bangkok Co., Ltd., 2006, 25.

[18] VisiLogicTM Ladder Programming: Software Manual, V230-21-G23 Rev: 12/04. http://d1.cie.put. poznan.pl/dav/zietkiew/VisiLogic_Software_Manual-Ladder.pdf. Accessed January 2015.

[19] Aydin Tözeren, Human Body Dynamics: Classical Mechanics and Human Movement, Springer Science \& Business Media, 2000. 


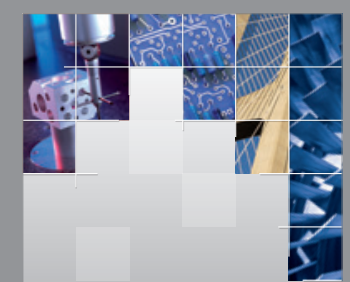

\section{Enfincering}
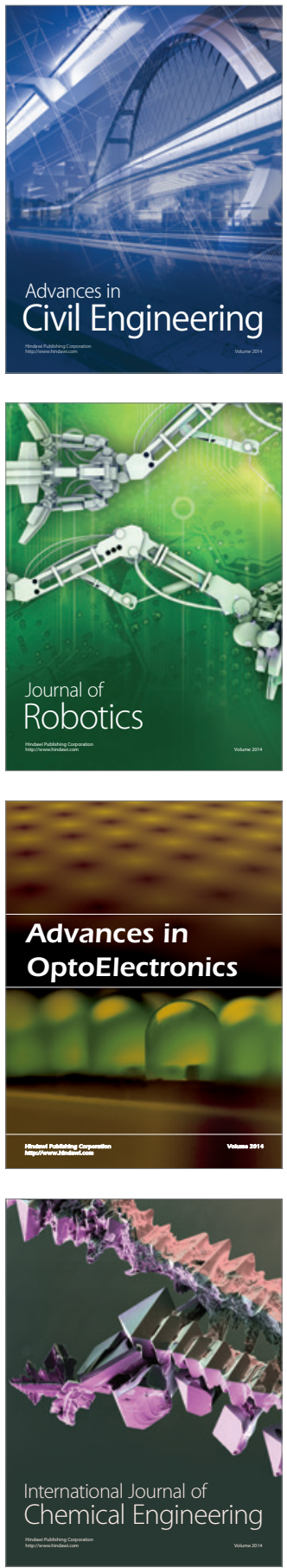

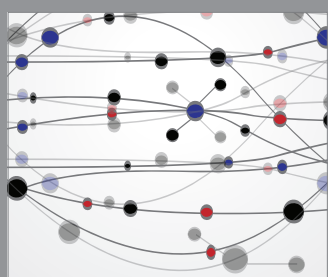

The Scientific World Journal

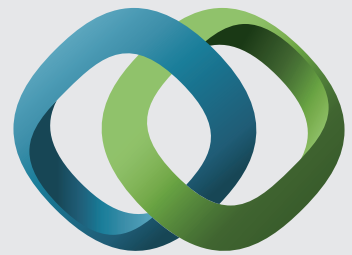

\section{Hindawi}

Submit your manuscripts at

http://www.hindawi.com
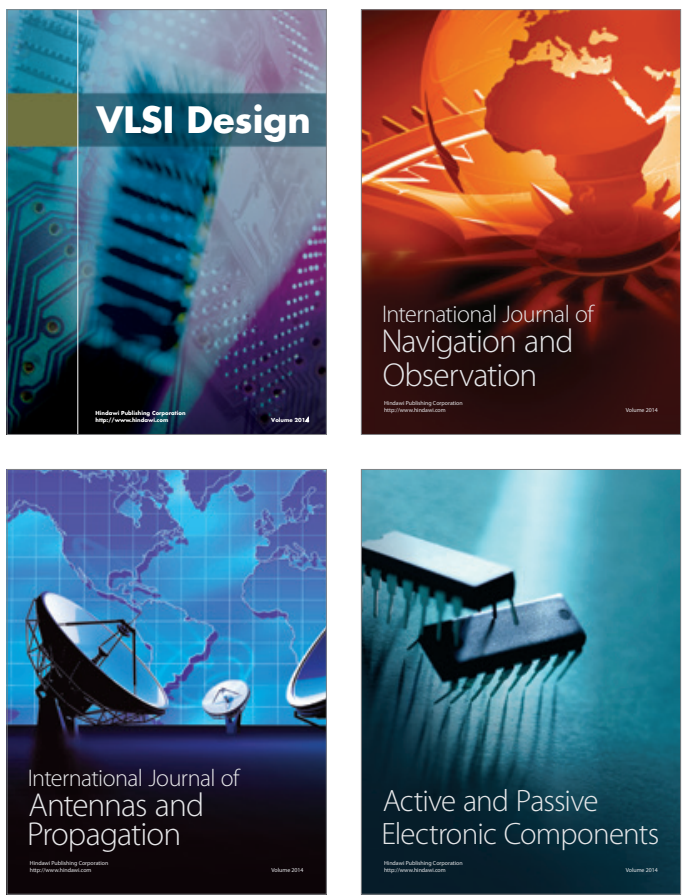
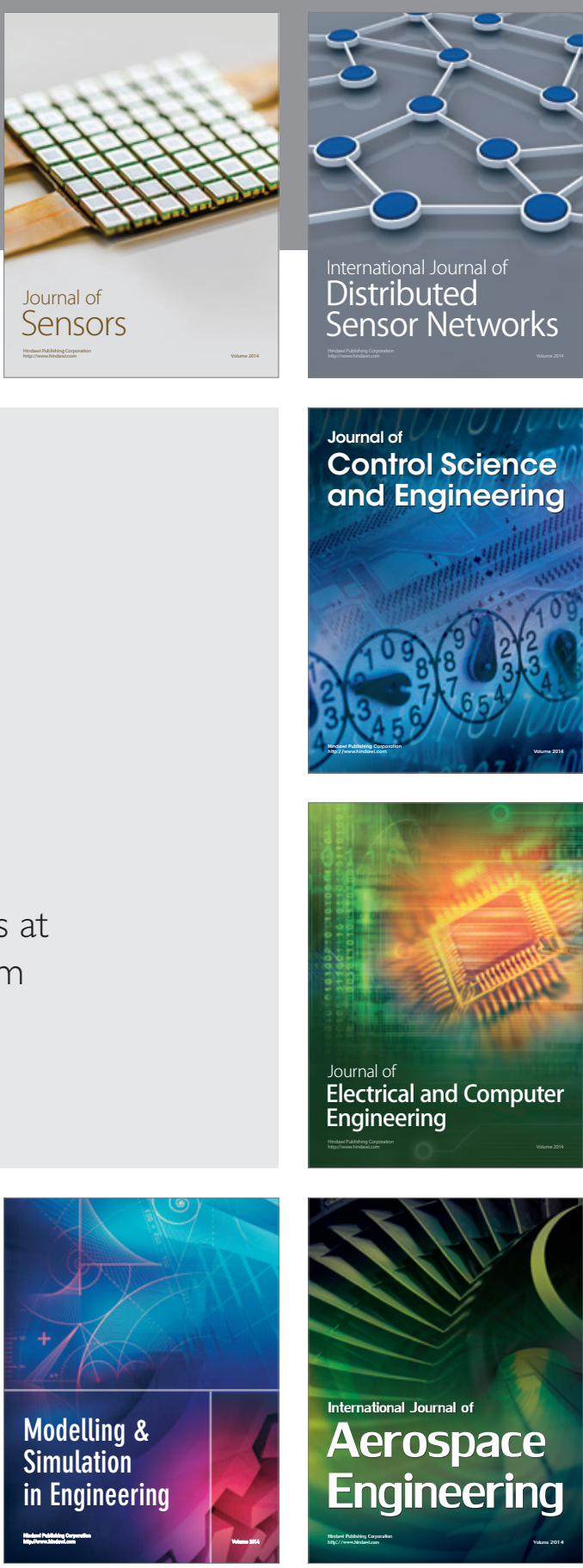

International Journal of

Distributed

Sensor Networks

Journal of

Control Science

and Engineering
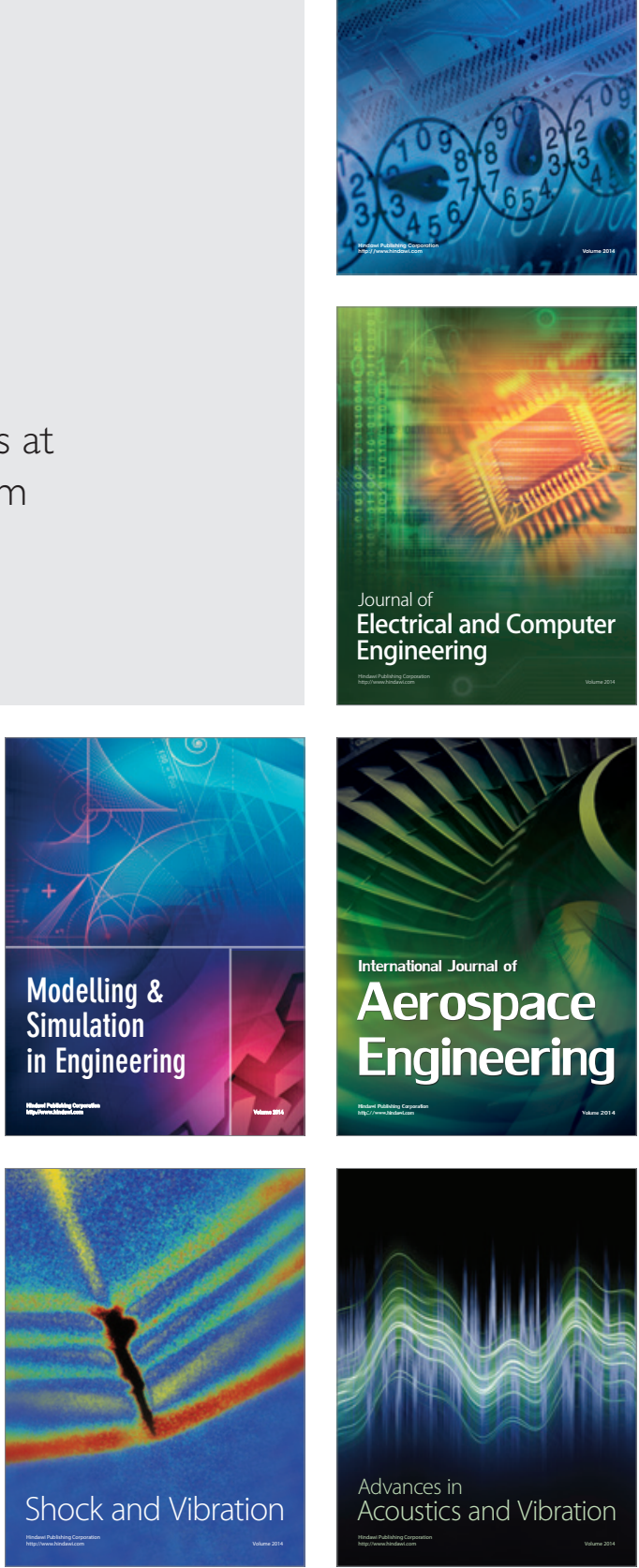